\title{
A macrocyclic receptor containing two viologen species connected by conjugated terphenyl groups
}

Article

Supplemental Material

Chen, L., Lim, K. J. C., Babra, T. S., Taylor, J. O., Pižl, M., Evans, R., Chippindale, A. M., Hartl, F., Colquhoun, H. M. and Greenland, B. W. (2018) A macrocyclic receptor containing two viologen species connected by conjugated terphenyl groups. Organic \& Biomolecular Chemistry, 16 (27). pp. 5006-5015. ISSN 1477-0520 doi: https://doi.org/10.1039/c8ob00919h Available at https://centaur.reading.ac.uk/78400/

It is advisable to refer to the publisher's version if you intend to cite from the work. See Guidance on citing.

Published version at: http://dx.doi.org/10.1039/c8ob00919h

To link to this article DOI: http://dx.doi.org/10.1039/c8ob00919h

Publisher: Royal Society of Chemistry

All outputs in CentAUR are protected by Intellectual Property Rights law, including copyright law. Copyright and IPR is retained by the creators or other copyright holders. Terms and conditions for use of this material are defined in the End User Agreement. 


\section{CentAUR}

Central Archive at the University of Reading

Reading's research outputs online 


\section{Supporting Information}

\section{A macrocyclic receptor containing two viologen species connected by}

\section{conjugated terphenyl groups}

Long Chen, ${ }^{a}$ Kate J. C. Lim, ${ }^{a}$ Tahkur S. Babra, ${ }^{a}$ James O. Taylor, ${ }^{a}$ Martin Pižl, ${ }^{b, c} \quad$ Robert Evans, ${ }^{d}$ Ann M. Chippindale, ${ }^{a}$ František Hartl, ${ }^{a}$ Howard M. Colquhoun*a and Barnaby W. Greenland*a,e

aDepartment of Chemistry, University of Reading, Whiteknights, Reading, RG6 6AD, UK; email: h.m.colquhoun@reading.ac.uk

${ }^{b}$ J. Heyrovský Institute of Physical Chemistry, Academy of Sciences of the Czech Republic, Dolejškova

3, CZ-182 23 Prague, Czech Republic

${ }^{c}$ Department of Inorganic Chemistry, University of Chemistry and Technology, Prague, Technická 5, CZ16628 Prague, Czech Republic

d Aston University Institute of Materials Research, School of Engineering and Applied Science, Birmingham, B4 7ET, UK.

e Department of Chemistry, School of Life Sciences, University of Sussex, Falmer, BN1 9QJ, UK; email b.w.greenland@sussex.ac.uk

Contents

Table S1 Atom numbering and comparison of structural data of $\mathbf{4}^{4+}$ from solid state analysis and DFT calculations.

Table S2 Selected DFT calculated bond lengths and angles for $\mathbf{4}^{4+}, \mathbf{4}^{2(\bullet+)}$ and 4

Table S3. TD-DFT (PBE0/PCM-MeCN) calculated low-lying transitions of triplet $\mathbf{4}^{2(\bullet+)}$ with oscillator strength higher than 0.005)..... S5

Figure S1 Molecular orbitals of $\mathbf{4}^{4+}$ involved in the calculated transition at $339 \mathrm{~nm}$.............S6

Figure S2 Molecular orbitals of $\mathbf{4}^{2(\bullet+)}$ involved in the calculated transition at $584 \mathrm{~nm}$...........S6

Figure S3 Molecular orbitals of $\mathbf{4}$ involved in the calculated transition at $453 \mathrm{~nm}$.................S7

Figure S4 Calculated electronic absorption spectra for $\mathbf{4}^{2(\bullet+)}$ with vertical excitations..........S7

Figure S5 Calculated $\alpha$-spinorbitals of $\mathbf{4}^{2(\bullet+)}$ involved in UV-vis electronic transitions............S8

Figure S6 Calculated b-spinorbitals of $\mathbf{4}^{2(\bullet+)}$ involved in UV-vis electronic transitions...........S9

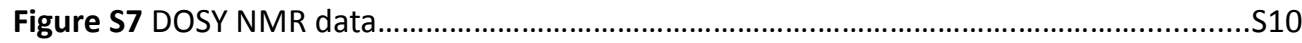

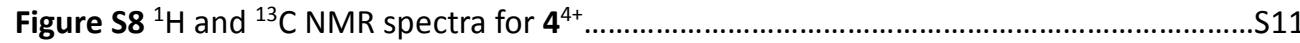

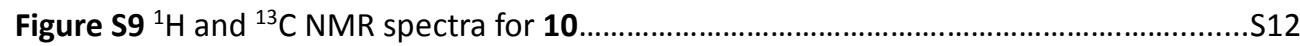


Table S1. A comparison of selected experimental and in vacuo DFT calculated bond lengths $(\AA)$ and angles $\left(^{\circ}\right)$ for $4^{4+}$.

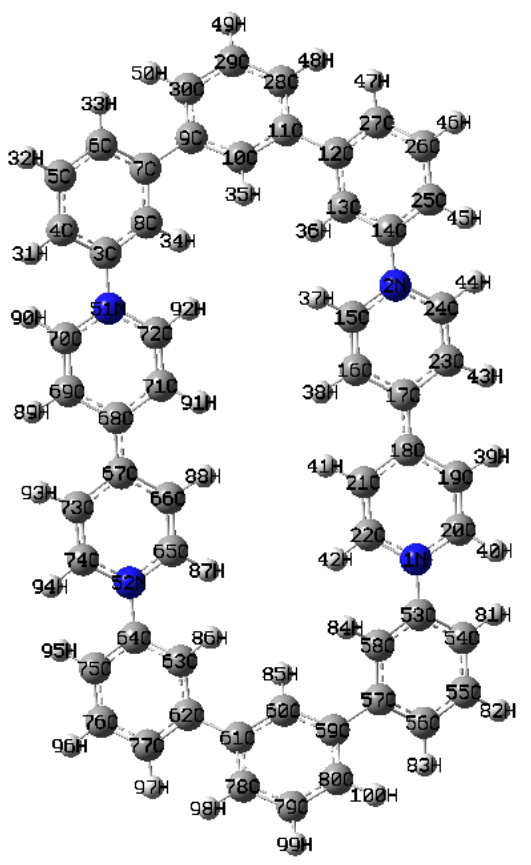

Macrocycle $\mathbf{4}^{4+}$ with atoms numbered.

\begin{tabular}{|c|c|c|}
\hline Bond & Exp. & Calc. \\
& & (in vacuo) \\
\hline N51-C3 & 1.451 & 1.447 \\
\hline N52-C64 & 1.450 & 1.447 \\
\hline N1-C53 & 1.451 & 1.447 \\
\hline N2-C14 & 1.450 & 1.447 \\
\hline N1-C22 & 1.353 & 1.349 \\
\hline C22-C21 & 1.375 & 1.374 \\
\hline C21-C18 & 1.368 & 1.398 \\
\hline C18-C17 & 1.470 & 1.484 \\
\hline C17-C16 & 1.397 & 1.398 \\
\hline C16-C15 & 1.355 & 1.374 \\
\hline C15-N2 & 1.352 & 1.349 \\
\hline N52-C65 & 1.352 & 1.349 \\
\hline C65-C66 & 1.355 & 1.374 \\
\hline C66-C67 & 1.397 & 1.398 \\
\hline C67-C68 & 1.470 & 1.484 \\
\hline C68-C71 & 1.368 & 1.398 \\
\hline C71-C72 & 1.375 & 1.374 \\
\hline C72-N51 & 1.353 & 1.349 \\
\hline & & \\
\hline & & \\
\hline
\end{tabular}

\begin{tabular}{|c|c|c|}
\hline Angle & Exp. & $\begin{array}{c}\text { Calc. } \\
\text { (in vacuo) }\end{array}$ \\
\hline N51-C3-C8 & 118.3 & 117.7 \\
\hline C3-N51-C72 & 117.8 & 118.5 \\
\hline C71-C68-C67 & 120.5 & 117.9 \\
\hline C68-C67-C66 & 120.6 & 117.9 \\
\hline C65-N52-C64 & 119.8 & 118.5 \\
\hline N52-C64-C63 & 117.8 & 117.7 \\
\hline N2-C14-C13 & 117.8 & 117.7 \\
\hline C14-N2-C15 & 119.8 & 118.5 \\
\hline C16-C17-C18 & 120.6 & 117.9 \\
\hline C17-C18-C21 & 120.5 & 117.9 \\
\hline C22-N1-C53 & 117.8 & 118.5 \\
\hline N1-C53-C58 & 118.3 & 117.7 \\
\hline & & \\
\hline & & \\
\hline & & \\
\hline & & \\
\hline & & \\
\hline & & \\
\hline & & \\
\hline & & \\
\hline & & \\
\hline & & \\
\hline & & \\
\hline & & \\
\hline & & \\
\hline
\end{tabular}


Table S2. Comparison of selected DFT calculated (polarizable continuum model, PCM-MeCN) bond lengths $(\AA)$ and angles $\left({ }^{\circ}\right)$ for $\mathbf{4}^{4+}, \mathbf{4}^{2(\cdot+)}$ and $\mathbf{4}$ (for atom numbering see Table $S 1$ ).

Note the strong variation in the $\mathrm{C}-\mathrm{N}$ and $\mathrm{C}-\mathrm{C}$ bond lengths in the heterocycle rings (red entries) for each of the sequential oxidation states of the macrocycle $\left(4^{4+}, 4^{2(0+)}\right.$ and 4$)$. In contrast, note the relative invariance with oxidation state of the bond lengths calculated in the polyaromatic spacer units (purple entries). The C-N bonds between the heterocycle rings and polyaromatic spacer units (green entries) also become shorter upon the reduction, which is ascribed to a better $\pi$-overlap between $C(p)$ and $N(p)$ orbitals due increasing planarity of the viologen units (Figure 6 in the main text).

\begin{tabular}{|c|c|c|c|}
\hline & \multicolumn{3}{|c|}{ Bond Length (̊) } \\
\hline Bond & $\mathbf{4}^{4+}$ & $\mathbf{4}^{2(\bullet+)}$ & $\mathbf{4}$ \\
\hline N51-C3 & 1.442 & 1.424 & 1.403 \\
\hline N52-C64 & 1.442 & 1.425 & 1.403 \\
\hline N1-C53 & 1.442 & 1.424 & 1.403 \\
\hline N2-C14 & 1.442 & 1.425 & 1.403 \\
\hline N1-C22 & 1.350 & 1.370 & 1.391 \\
\hline C22-C21 & 1.373 & 1.358 & 1.346 \\
\hline C21-C18 & 1.395 & 1.422 & 1.448 \\
\hline C18-C17 & 1.473 & 1.423 & 1.381 \\
\hline C17-C16 & 1.395 & 1.422 & 1.448 \\
\hline C16-C15 & 1.373 & 1.358 & 1.346 \\
\hline C15-N2 & 1.349 & 1.370 & 1.390 \\
\hline N52-C65 & 1.349 & 1.370 & 1.390 \\
\hline C65-C66 & 1.373 & 1.358 & 1.346 \\
\hline C66-C67 & 1.395 & 1.422 & 1.448 \\
\hline C67-C68 & 1.473 & 1.423 & 1.381 \\
\hline C68-C71 & 1.395 & 1.422 & 1.448 \\
\hline C71-C72 & 1.373 & 1.358 & 1.346 \\
\hline C72-N51 & 1.350 & 1.370 & 1.391 \\
\hline C3-C8 & 1.386 & 1.390 & 1.397 \\
\hline C8-C7 & 1.395 & 1.395 & 1.395 \\
\hline C7-C9 & 1.476 & 1.476 & 1.477 \\
\hline C9-C10 & 1.395 & 1.394 & 1.395 \\
\hline C10-C11 & 1.394 & 1.394 & 1.395 \\
\hline C11-C12 & 1.476 & 1.476 & 1.477 \\
\hline C12-C13 & 1.395 & 1.395 & 1.395 \\
\hline C13-C14 & 1.386 & 1.389 & 1.397 \\
\hline C53-C58 & 1.386 & 1.390 & 1.397 \\
\hline C58-C57 & 1.395 & 1.395 & 1.395 \\
\hline C57-C59 & 1.476 & 1.476 & 1.477 \\
\hline C59-C60 & 1.395 & 1.394 & 1.395 \\
\hline C60-C61 & 1.394 & 1.394 & 1.395 \\
\hline & & & \\
\hline
\end{tabular}

\begin{tabular}{|c|c|c|c|}
\hline & \multicolumn{3}{|c|}{ Bond Angle ( ${ }^{\circ}$ ) } \\
\hline & $\mathbf{4}^{4+}$ & $\mathbf{4}^{2(\bullet+)}$ & $\mathbf{4}$ \\
\hline N51-C3-C8 & 118.2 & 119.1 & 120.1 \\
\hline C3-N51-C72 & 119.2 & 120.4 & 121.4 \\
\hline C71-C68-C67 & 120.2 & 122.2 & 123.4 \\
\hline C68-C67-C66 & 120.1 & 122.2 & 123.4 \\
\hline C65-N52-C64 & 119.1 & 120.4 & 121.3 \\
\hline N52-C64-C63 & 118.3 & 119.1 & 120.0 \\
\hline N2-C14-C13 & 118.3 & 119.1 & 120.0 \\
\hline C14-N2-C15 & 119.1 & 120.4 & 121.3 \\
\hline C16-C17-C18 & 120.1 & 122.2 & 123.4 \\
\hline C17-C18-C21 & 120.1 & 122.2 & 123.4 \\
\hline C22-N1-C53 & 119.1 & 120.4 & 121.4 \\
\hline N1-C53-C58 & 118.2 & 119.1 & 120.1 \\
\hline
\end{tabular}




\begin{tabular}{|l|l|l|l|}
\hline C61-C62 & 1.476 & 1.476 & 1.477 \\
\hline C62-C63 & 1.395 & 1.395 & 1.395 \\
\hline C63-C64 & 1.386 & 1.389 & 1.397 \\
\hline
\end{tabular}


Table S3. TD-DFT (PBEO/PCM-MeCN) calculated low-lying transitions of triplet $\mathbf{4}^{2\left({ }^{++}\right)}$with oscillator strength higher than 0.005 (see Figure S7). Molecular spinorbitals involved in the electronic transitions are depicted in Figures S8 and S9.

\begin{tabular}{|c|c|c|}
\hline Main component (\%) & $\begin{array}{c}\text { Transition } \\
\text { energy [eV] } \\
(\mathrm{nm})\end{array}$ & $\begin{array}{l}\text { Oscillator } \\
\text { strength }\end{array}$ \\
\hline 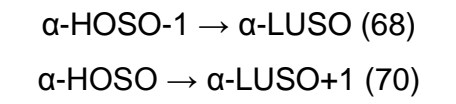 & $2.04(607)$ & Weak $(\sim 0)$ \\
\hline 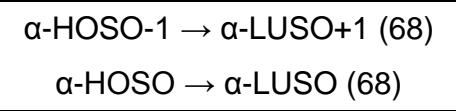 & $2.12(584)$ & 1.090 \\
\hline 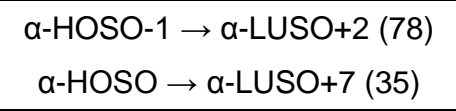 & $2.88(431)$ & 0.014 \\
\hline$\alpha$-HOSO $\rightarrow \alpha$-LUSO+3 (71) & $2.90(428)$ & 0.137 \\
\hline $\begin{array}{l}\alpha \text {-HOSO } \rightarrow \alpha \text {-LUSO+3 (40) } \\
\beta \text {-HOSO-2 } \rightarrow \beta \text {-LUSO (38) } \\
\beta \text {-HOSO } \rightarrow \beta \text {-LUSO+1 (49) }\end{array}$ & $2.98(416)$ & 0.942 \\
\hline$\beta$-HOSO-1 $\rightarrow \beta$-LUSO (62) & $3.15(394)$ & 0.035 \\
\hline$\beta$-HOSO $\rightarrow \beta$-LUSO+1 (44) & $3.16(392)$ & 0.315 \\
\hline$\beta$-HOSO-1 $\rightarrow \beta$-LUSO (64) & $3.31(374)$ & 0.006 \\
\hline $\begin{array}{c}\beta \text {-HOSO } \rightarrow \beta \text {-LUSO+1 (65) } \\
\beta \text {-HOSO-4 } \rightarrow \beta \text {-LUSO+1 (45) } \\
\beta \text {-HOSO-5 } \rightarrow \beta \text {-LUSO (39) }\end{array}$ & $3.33(373)$ & 0.439 \\
\hline $\begin{array}{c}\beta \text {-HOSO-2 } \rightarrow \beta \text {-LUSO (76) } \\
\beta \text {-HOSO-5 } \rightarrow \beta \text {-LUSO (41) } \\
\beta \text {-HOSO-4 } \rightarrow \beta \text {-LUSO+1 (40) }\end{array}$ & $3.54(351)$ & 0.058 \\
\hline 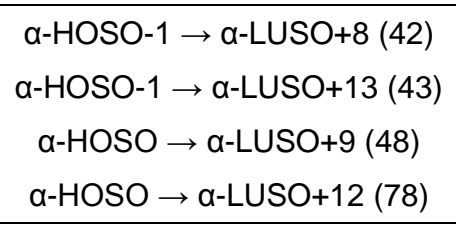 & $3.60(344)$ & 0.029 \\
\hline 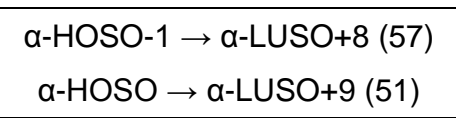 & $3.61(343)$ & 0.020 \\
\hline $\begin{array}{c}\alpha \text {-HOSO-1 } \rightarrow \text { a-LUSO+10 (78) } \\
\alpha \text {-HOSO } \rightarrow \alpha \text {-LUSO+11 (44) } \\
\beta \text {-HOSO-3 } \rightarrow \beta \text {-LUSO+1 (40) }\end{array}$ & $3.62(342)$ & 0.013 \\
\hline $\begin{array}{c}\beta \text {-HOSO-7 } \rightarrow \beta \text {-LUSO (54) } \\
\beta \text {-HOSO-6 } \rightarrow \beta \text {-LUSO+1 (54) }\end{array}$ & $3.83(324)$ & 0.13 \\
\hline Mixed & $3.98(312)$ & 0.024 \\
\hline $\begin{array}{c}\beta \text {-HOSO-12 } \rightarrow \beta \text {-LUSO (59) } \\
\beta \text {-HOSO-11 } \rightarrow \beta \text {-LUSO+1 (59) }\end{array}$ & $3.99(311)$ & 0.13 \\
\hline $\begin{array}{c}\beta \text {-HOSO-13 } \rightarrow \beta \text {-LUSO+1 (66) } \\
\beta \text {-HOSO-14 } \rightarrow \beta \text {-LUSO (67) }\end{array}$ & $4.06(305)$ & 0.090 \\
\hline
\end{tabular}




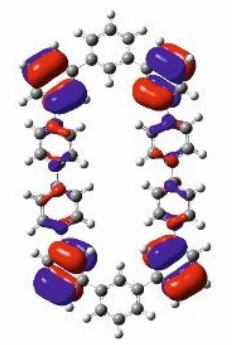

HOMO-5

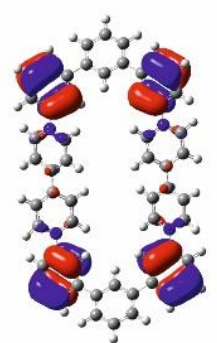

HOMO-6

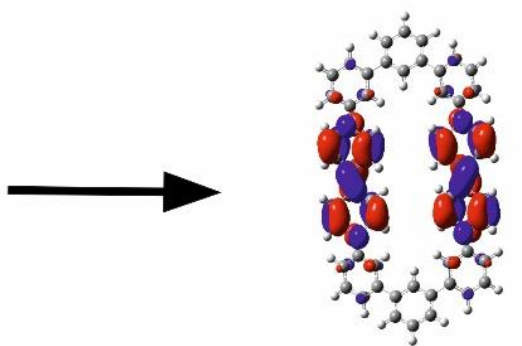

LUMO

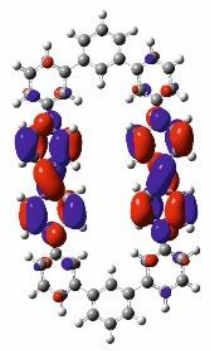

LUMO+1

Figure S1. Molecular orbitals of $\mathbf{4}^{4+}$ involved in the calculated transition at $339 \mathrm{~nm}$.

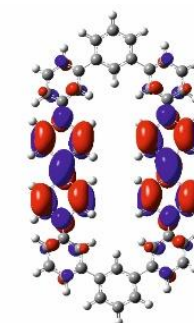

$\alpha$-HOSO-1

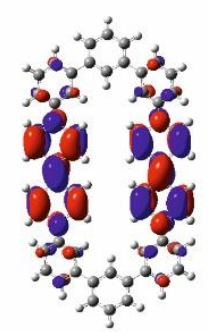

$\alpha-\mathrm{HOSO}$

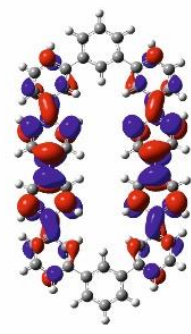

$\alpha$-LUSO+1

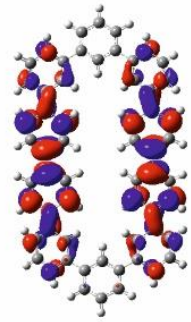

$\alpha$-LUSO

Figure S2. Molecular orbitals of $4^{2(\bullet+)}$ involved in the calculated transition at $584 \mathrm{~nm}$. 


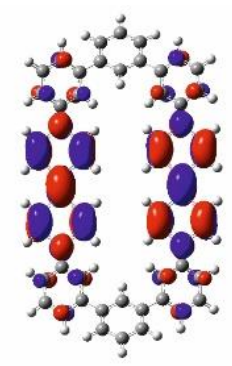

HOMO

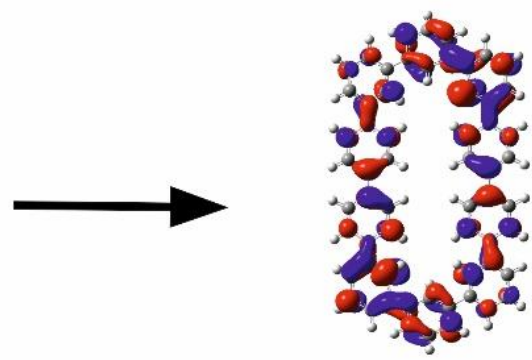

LUMO+3

Figure S3. Molecular orbitals of 4 involved in the calculated transition at $453 \mathrm{~nm}$.

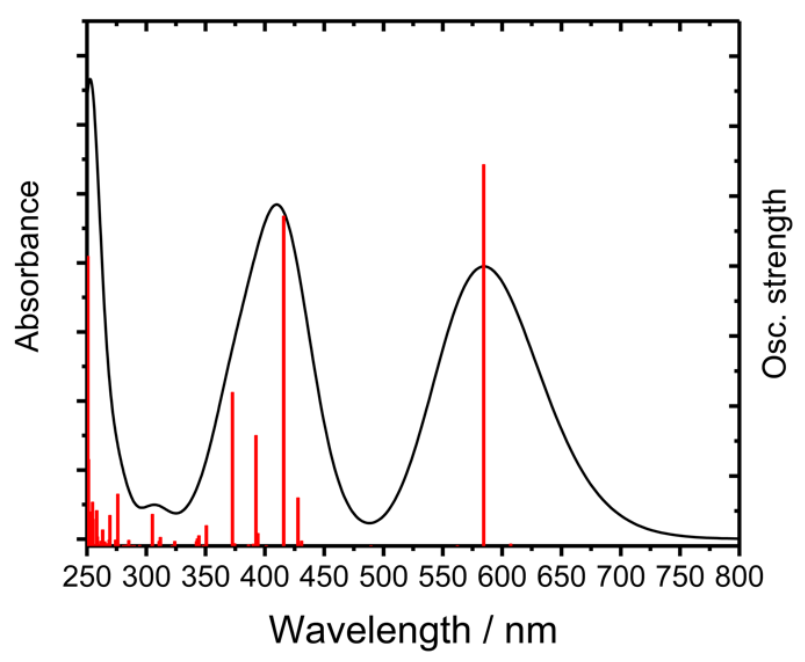

Figure S4. Calculated electronic absorption spectra for $\mathbf{4}^{2(\bullet+)}$ with vertical excitations. (See Table S3 and Figures S5 and S6 for more details.) 

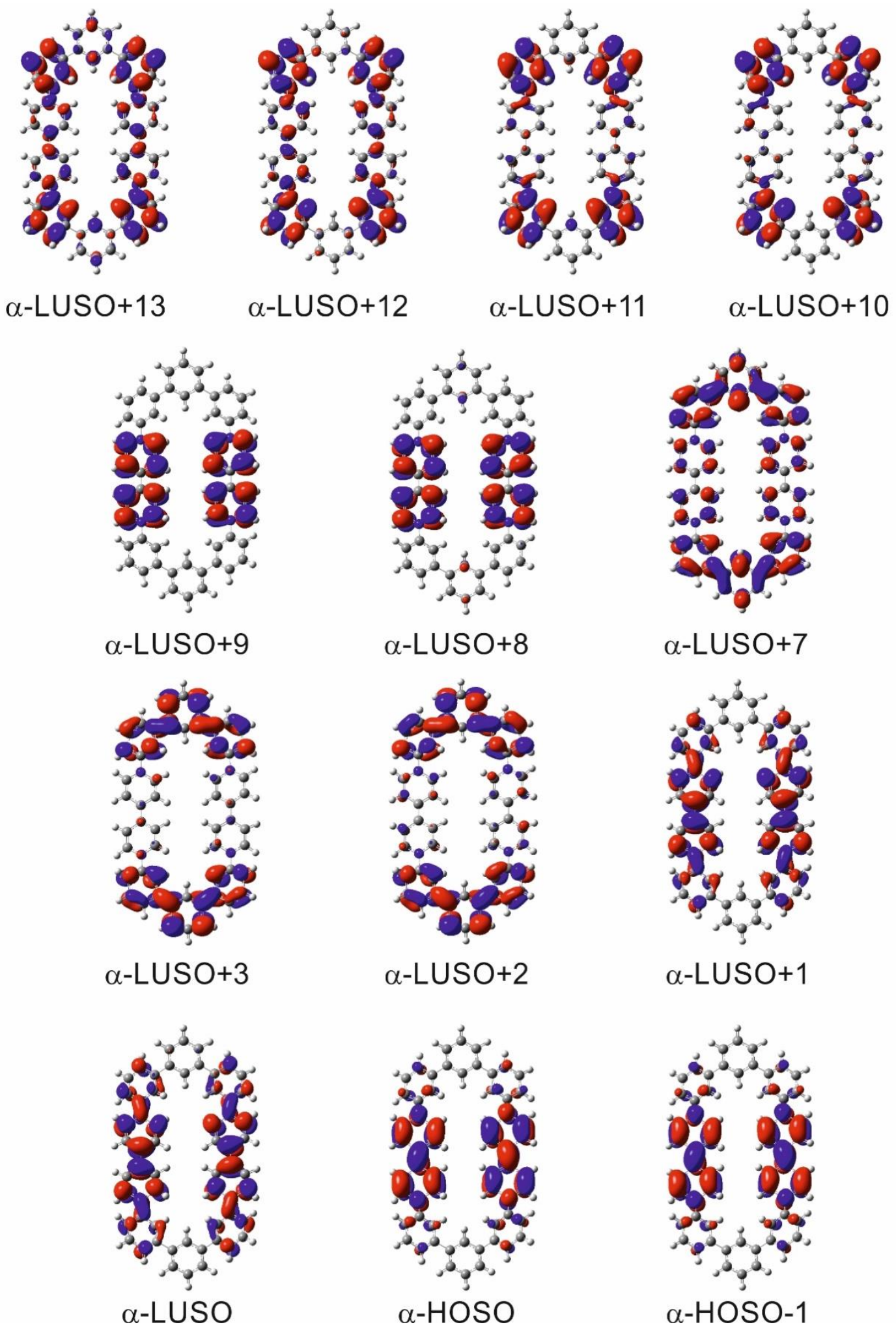

Figure S5. Calculated $\alpha$-spinorbitals of $4^{2(\cdot+)}$ involved in the UV-vis electronic transitions (Table S3). 

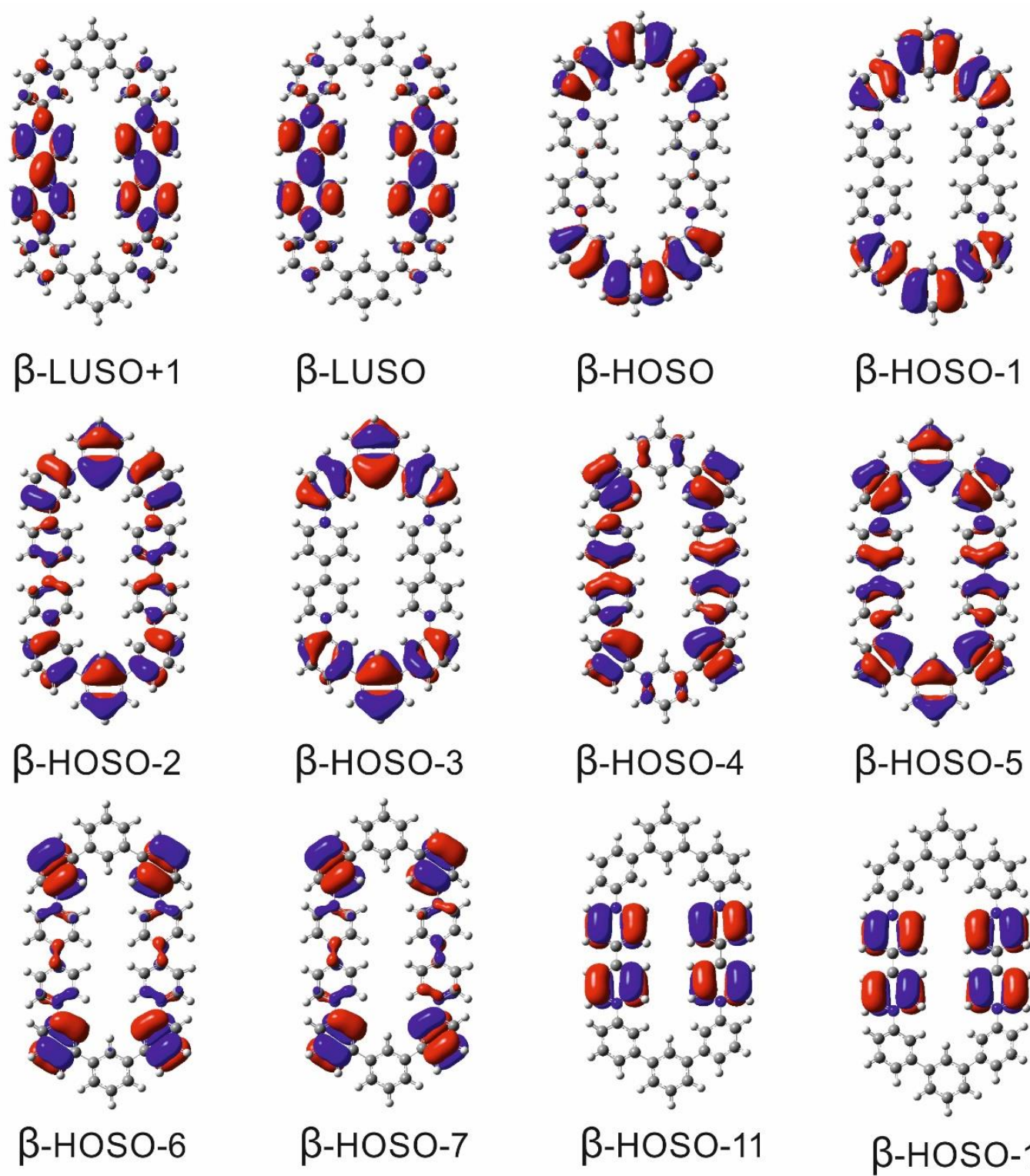

$\beta$-HOSO-5
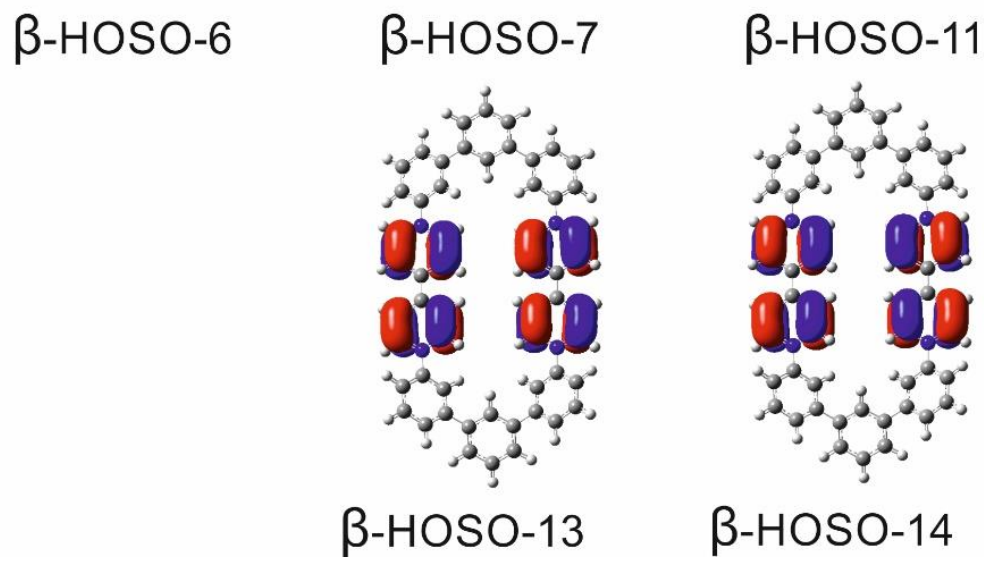

$\beta$-HOSO-13 $\quad \beta$-HOSO-14

Figure S6. Calculated $\beta$-spinorbitals of $\mathbf{4}^{2(\bullet+)}$ involved in the UV-vis electronic transitions (Table S3). 


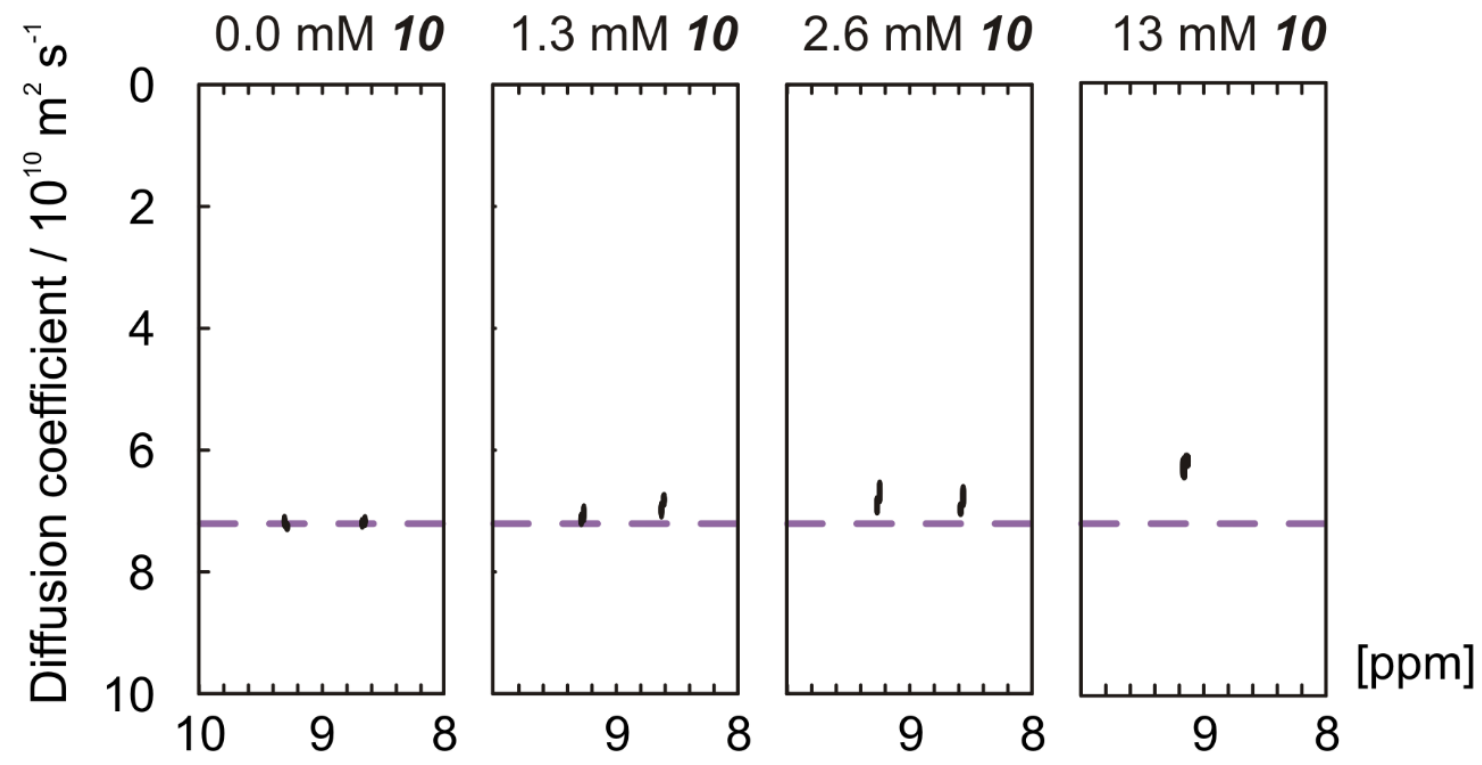

Figure S7. Four DOSY spectra $\left(\mathrm{CD}_{3} \mathrm{CN}\right)$ corresponding to samples of $1.3 \mathrm{mM}$ $4^{4+}$ and $0,1.3,2.6$ and $13 \mathrm{mM} 10$ respectively (used to construct Figure 7 in the paper). Only the region between 8 and $10 \mathrm{ppm}$ shown. In the sample containing $13 \mathrm{mM} \mathrm{4}^{4+}$, peaks at ca. $8.5 \mathrm{ppm}$ are not included in analysis due to overlap of signals from both species. Purple line indicates estimate for diffusion coefficient of $\mathbf{4}^{4+}$ in the absence of any complexation. 
${ }^{1} \mathrm{H}$ and ${ }^{13} \mathrm{C}$ NMR spectra of $4^{4+}$
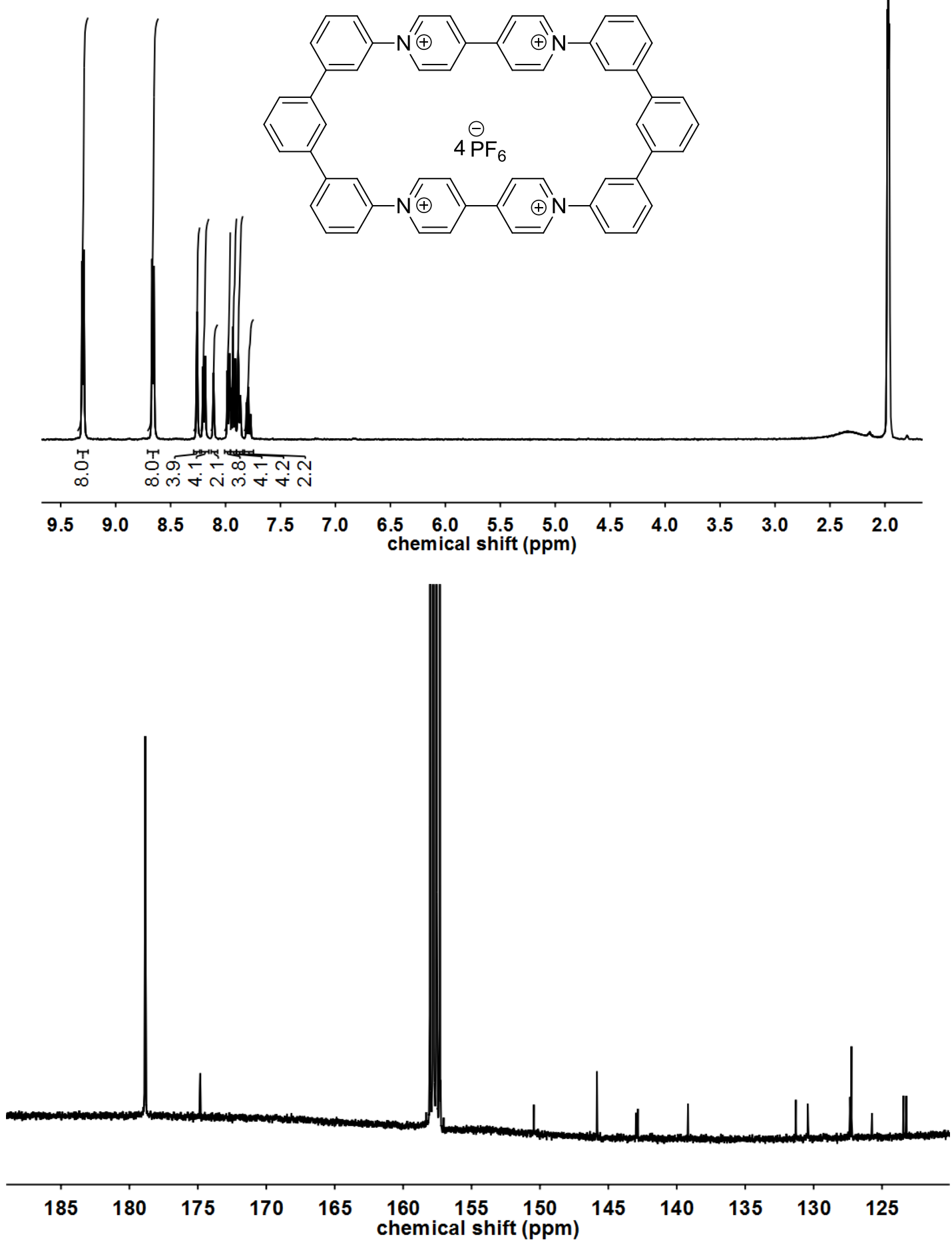

Figure S8. ${ }^{1} \mathrm{H}$ NMR $\left[\left(\mathrm{CD}_{3}\right)_{2} \mathrm{CO}, 400 \mathrm{MHz}\right]$ and ${ }^{13} \mathrm{C} \mathrm{NMR}\left(\mathrm{CD}_{3} \mathrm{CN}, 100 \mathrm{MHz}\right)$ spectra of $4^{4+} .4\left(\mathrm{PF}_{6}{ }^{-}\right)$ 
${ }^{1} \mathrm{H}$ and ${ }^{13} \mathrm{C}$ NMR spectra of $\mathbf{1 0}$
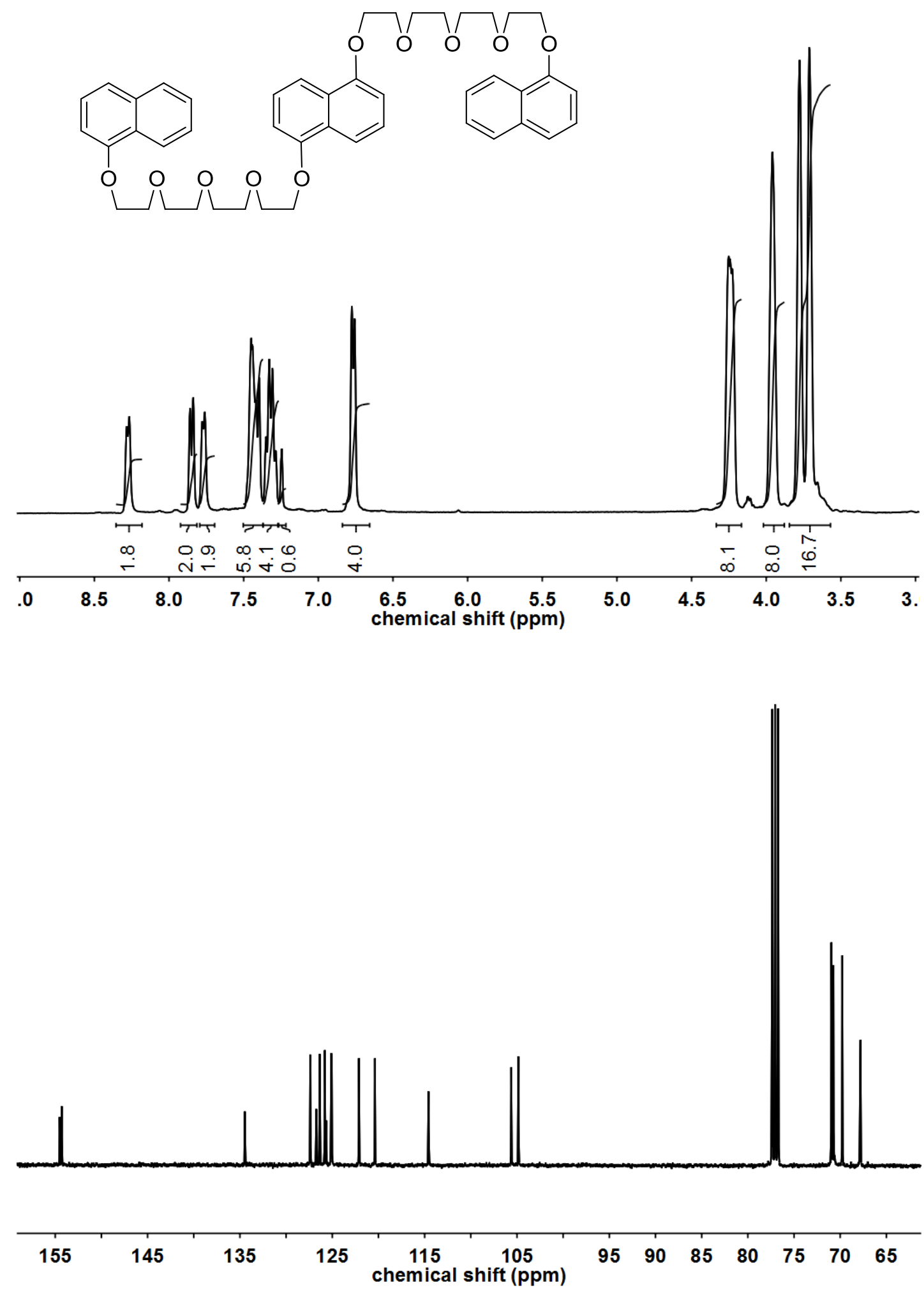

Figure S9. $400 \mathrm{MHz}{ }^{1} \mathrm{H}$ and $100 \mathrm{MHz}{ }^{13} \mathrm{C}$ NMR spectra of $10\left(\mathrm{CDCl}_{3}\right)$ 